\title{
Contents, Vol. 79, 1932
}

Inhaltsverzeichnis.

Eigenarbeiten. Seite

Bartels. M., Fixatorschalen zur Transplantation gegen Symble-

pharon 29

Bendescu, T., Beiderseitige Optikusatrophie , verursacht durch

Pneumosinus dilatans der rechten Keilbeinhöhle 41

Best, F., Zur Methodik der Gesichtsfelduntersuchung $\quad 457$

Bunge, E., Klinische Beiträge zur Pathologie der Linse (Hierzu

Tafel I/II) 5

Comberg, Ẁ., Ein neuer elektrischer Handaugenspiegel zur wahlweisen Benutzung für umgekehrtes und aufrechtes Bild . . 355 Fischer, Fr., Beitrag zu den Fehlbildungen des Auges (Netzhaut-

arterienschlinge, Linsenektopie) 158

Gasteiger, H., Zur Kenntnis der Keratokonusspaltlinien

Gebb, H., Das Pantokain in der Augenheilkunde 467

Grósz, E. v., Über das Trachom 470

Grüter, W'., Eine Pilzgeschwulst (Aktinomykose) im oberen Tränen-

röhrchen (Actinomyces discofoliatus) 477

Haas, E., und Schmidt, K., Zur Frage der nichtoperativen Behand-

lung der Neuritis retrobulbaris 449

Halåsz, C., Über Wanderung und Spontanausstoßung intraokularer

Kupfersplitter ... 33

Hamburg, J., Über günstige Beeinflussung der tabischen Sehnervenatrophie durch Steigerung der Zelloxydation 331

Herrenschwand, F.v., Über eine metastatischePilzinfektion in einem

Auge zehn Jahre nach Extraktion einer Katarakt bei Hetero-

chromie mit Zyklitis 223

Hesse, R., Über einen eigenartigen Glaskörperbefund. (Hierzu

Tafel V) 247

--, -, Über die anatomischen Grundlagen der v. Hippelschen

Netzhauterkrankung. (Hierzu Tafel V) 252

Hofe, K.vom, DieHäufigkeit von Augensymptomen, insbesondere

Störungen oder Pupillenreaktionen nach syphilitischer Infektion 511 Jungraw, J., Ein tödlich verlaufener Fall von Endophthalmitis

und Meningitis purulenta nach einer Messerverletzung der

Hornhaut

368

Kern, R., Ein Fall von kavernösem Hämangiom der Chorioidea 21 Kochmann, M., und Nabe, J., Quantitativ vergleichende Unter-

suchungen über die Wirkung der Lokalanästhetika auf die 
Hornhaut des Kaninchens

Krassó, I. und Strisower, R., Über die Wirkung des Karotisdruckversuches auf Blutdruck und Augendruck 172

Lauterstein, M., Ophthalmopathia trigeminociliaris. Einiges über die sogenannte Conjunctivitis chronica simplex und Conjunctiva neuroallergica . . 370

Lewi, M., Über umschriebene Kalkherde und Nekrosen in der

Lederhaut 180

Lindner, K., Über die Verwendung der Lochbrille bei Behandlung von Netzhautabhebungen . . . 191

Löhlein, W., Beitrag zur Ätiologie des „Glaukoms der Jugendlichen” 524 Meller, J , Die sympathisçhe Ophthalmie durch Gewebskultur aus dem sympathisierenden Auge als eine echte bazilläre Erkrankung nachgewiesen. (Hierzu Tafel III) 95

,-- , Über Bazillämie und sympathisçhe Ophthalmie no

- , -, Zur Kenntnis der pathologischen Histologie der spontanen disseminierten Chorioiditis 128

Nabe, J'., siehe Kochmann, M.

Perwög, M., Über die Tenotomie beider Recti interni in einer

Sitzung beim Strabismus convergens 239

Peters, A., Bemerkungen zur Entstehung einiger Starformen . . 1

Pillat, A., Über Mumifizierung des Hornhautepithels bei Vitamin-

A-Mangel. (Hierzu Tafel IV) 200

- IV

Seite

Riedl, F., Ein Beitrag zur Anatomie der Ablösung der Vorder-kapsellamelle, der sog.

„,Feuerlamelle”, bei Schmiede- undGlasmacherstar .... 287

Riegel, E., und Vogelsang, K., Über zentrales Skotom bei Chiasma-

erkrankungen 433

Sallmann, L., Über Augenveränderungen bei menschlicher Chrysosis

nach Sanocrysinbehandlung 208

Schmidt, K., Untersuchungen über die Veränderung der Gefäß-wanddurchlässigkeit bei enukleierten Kaninchenaugen und ein weiterer Versuch zur Bestimmung der absoluten Blutmenge im Kaninchenauge mit radioaktiven Indikatoren . . 427

,-- , siehe Haas, E.

Schneider, R., Über die dem Keratokonus entgegengesetzte Horn-hautkrümmung nebst Bemerkungen zum Formproblem desAugapfels. Ein Beitrag zur Arbeit Salzmanns über denKeratokonus 265

Seefelder, R., Über Drucksteígerung nach Nachstardurchschneidung nebst Bemerkungen über die nach Augenoperationen auf-tretenden Randinfiltrate und Randgeschwüre der Hornhaut 212

Stern, H., Über Entoptoskopie und ihre klinische Verwendbarkeit 361

Strisower, R., siehe Krassó, I.

Székely, J., Tuberkulose alsAtiologie der $1 \frac{1}{2} 2 \mathrm{e}^{7 / 8 s s c h e n ~ H e t e r o c h r o m i e ~} 272$

Urbanek, J., Zur Frage der Entstehung und Ätiologie der perio-

disch rezidivierenden Hypopyon-Üveitis 145

Vogelsang, K., siehe Riegel, Ë. 
Wegner, W'., Zur Kontrastuntersuchung der Tränenorgane mittels Thoriumdioxyd. (Mit einer Bemerkung über die Verwen-dung von Thoriumdioxyd zur Darstellung von Milz undLeber) 532 Berichte über die ophthalmologische Literatur.

Aderhaut. Netzhaut. Von Priv.-Doz. Dr. W. Riehm in Würzburg 50

Sehbahn, Auge und Nervenkrankheiten. Von Priv.-Doz. Dr. W.

Kyrieleis in Hamburg 302

Normale und pathologische Anatomie. Von Priv.-Doz. Dr. R.Schneider in Graz

374

Entwicklungsgeschichte des Auges. Mißbildungen. Anthropologie.

Von Prof. Dr. R. Seefelder in Innsbruck 386

Geschichte der Ophthalmologie. Von Prof. Dr. R. Seefelder in Innsbruck

Physiologische und Psychologische Optik. Von Priv.-Doz. Dr.

H. Gasteiger in Innsbruck 554

Vererbung und Auge. Von Priv.-Doz. Dr. A. Franceschetti in Basel 557

Gesellschaftsberichte.

Ophthalmolog. Gesellschaft in Wien. Sitzung vom 17. Oktober 1932403

Sitzung vom 21. November 1932575 Bericht über die 61. Versammlung des Vereins rheinischwestfälischer

Augenärzte am 17. April 1932 in Köln a. Rh 77

Schweizerische Ophthalmologische Gesellschaft. 25. Jahresver-

sammlung am 25. und 26. Juni 1932 in Biel 82, 420

Bericht über die X. Tagung der Nordwestdeutschen Augenärzt-

lichen Gesellschaft in Rostock am 23. und 24. Juli 1932 . 410

Diagnose und Therapie $\quad 91,326,420$

Buchbesprechungen 94, 330, 426, 580

P. Personalien 94

426

580

Das Sach- und Namenregister zu diesem Bande erscheint in Band 80 als gemeinsames Register fur diese beiden Bände. 